\title{
Déficits immunitaires liés à un défaut d'expression du récepteur pour l'antigène
}

Le lymphocyte $\mathrm{T}$ est au centre de la réponse immunitaire de type cellulaire. Il reconnaît l'antigène peptidique présenté par le complexe majeur d'histocompatibilité, par l'intermédiaire d'un complexe membranaire: le récepteur T/CD3.

Le complexe TCR/CD3 est composé : (1) de deux chaînes polymorphes ct glycosylées TCR- $\alpha$ et $-\beta$ (ou $-\gamma$ et $-\delta$ ) reliées par un pont disulfure et impliquées directement dans la reconnaissance de l'antigène par leur partic variable ; (2) du complexe CD3, associé de façon non covalente, constitué de trois chaînes monomorphes : $\gamma, \delta$ et $\epsilon ;$ (3) d'un homodimère $\zeta-\zeta$ ou hétérodimère $(\zeta-\eta)$ qui s'associe finalement au complexe pour son expression membranaire (revuc dans [1]). Tous les gènes codant pour les différentes protéines du complexe ont été clonés. Les gènes codant pour les protéines CD3- $\gamma,-\delta$ et $-\epsilon$ sont situés dans une même région chromosomique: la bande q23 du chromosome 11. Le clonage de ces gènes a montré une très grande homologie entre les gènes CD3- $\gamma$ et CD3- $\delta$; séparés seulement par une région non codante de 1,6 kb, lcurs sens de transcription sont inversés. Cela permet de penser que ces deux gènes proviennent de la duplication d'un même gène. Le gène codant pour la chaîne CD3- $\epsilon$ est moins homologue aux gènes CD3- $\gamma$ et $-\delta$, et est situé à $30 \mathrm{~kb}$ en aval. Il est composé de neuf exons, dont un scul code pour la partic transmembranaire de la protéine, et s'étend sur $12 \mathrm{~kb}$ (revue dans [2]). Les chaînes $\zeta$ et $\eta$ sont génétiquement distinctes des chaînes du CD3. Elles proviennent d'un épissage différentiel d'un même locus [3] et sont localisées sur le chromosome 1q.

Les chaînes du complexc CD3 et la chaîne $\zeta$ sont impliquées dans la trans-

comme les réccpteurs pour les $\mathrm{IgE}$, une région consensus dans leur partie cytoplasmique. Les complexes CD3 et $\zeta$ constituent deux modules autonomes de transduction des signaux. En effet, chaque module est capable de déclencher un événement tardif de l'activation cellulaire comme la production d'IL-2. Cependant ces deux modules ne sont pas totalement échangeables et chacun possc̀de des caractéristiques propres [4].

L'analyse de mutants expérimentaux déficients pour l'expression du complexe TCR/CD3 a montré que seuls les complexes composés de toutes les chaînes sont exprimés à la surface du lymphocytc T [5]. Blumberg et al. ont proposé la co-existence, à la surface membranaire, de complexes distincts composés des chaînes $\alpha-\beta$ du TCR ct CD3- $\gamma \epsilon$ ou CD3- $\delta \epsilon$ [6]. Ces auteurs ont aussi démontré la présence de deux chaînes CD3- $\epsilon$ par complexe et ces résultats ont été confirmés par d'autres études [7]. L'étude d'une lignée TCR/CD3 déficiente a permis de montrer qu'il peut exister, à la surface, des complexes TCR/CD3 dont la chaîne CD3- $\gamma$ est absente. De telles cellules peuvent être stimulées par des antigènes ou par d'autres stimuli du TCR [8]. Cette étude confirme l'hypothèse de flexibilité d'expression de complexes de type CD3- $\gamma \epsilon$ ou CD3- $\delta \epsilon$. Toutefois, cette flexibilité ne semble pas être partagée dans toutes les espèces car une lignée T murine, caractérisée par la délétion du gène codant pour la chaîne CD3- $\delta$, n'exprime pas à la surface de complexe de type CD3- $\gamma$ [9]. La chaîne CD3- $\epsilon$ est nécessaire à l'expression des deux types de complexe à la surface membranaire du lymphocyte $\mathrm{T}$ car elle stabilise les souscomplexes $\gamma-\epsilon$ et $\delta-\epsilon[10]$. L'assemblage du complexe a lieu dans le réticulum endoplasmique et, très tôt au cours de la biosynthèse, une protéine de $28 \mathrm{kDa}(\omega)$ s'associe aux complexes partiellement assemblés et aux chaînes $\alpha$ et $\beta$ du TCR. Cette protéine est impliquée dans le contrôle de l'assemblage intracellulaire en stabilisant l'association de complexes partiels ou en dirigeant les complexes incorrectement assemblés vers un compartiment où ils seront dégradés [11]. Après la glycosylation, les complexes sont exprimés à la surface du lymphocyte $T$. Les protéines en excès non assemblées sont rapidement et efficacement dégradées [12].

Les déficits immunitaires héréditaires regroupent des maladies très diverses responsables d'infections plus ou moins sévères et récurrentes. Parmi les déficits immunitaires des lymphocytes $\mathrm{T}$, et dont, pour la plupart, les bases moléculaires n'ont pas été élucidées, seulement deux déficits liés à un défaut d'expression du récepteur pour l'antigc̀ne ont été décrits à ce jour.

Nous avons étudié le déficit immunitaire d'un patient de cinq ans qui présente un défaut d'expression membranaire du complexe TCR/CD3. L'analyse en cytofluorimétrie des lymphocytes sanguins révèle que l'intensité d'expression du complexe TCR/CD3 par les lymphocytes, par ailleurs présents en nombre normal, n'atteint qu'un dixième de l'intensité attendue.

L'étude des ARN messagers codant pour les différentes chaînes du CD3 a montré que le transcrit de la chaîne CD3- $\epsilon$ était présent, mais en quantité réduite et de taille anormale [13]. Cela a conduit à rechercher des anomalies du gène de structure de la chaîne CD3- $\epsilon$. Le produit de PCR spécifique de la chaîne CD3- $\epsilon$ obtenu chez le patient présente une délétion complète de l'exon 7 qui conserve le cadre de lecture. Cet exon code pour la partie 


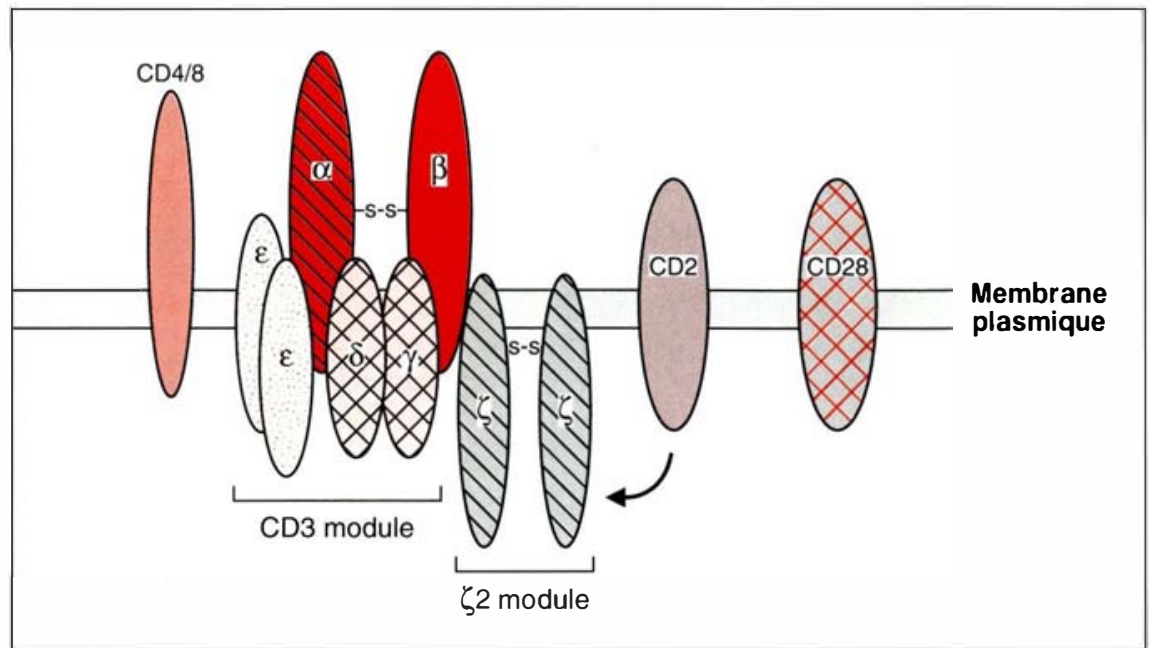

Figure 1. Représentation schématique du récepteur pour l'antigène à la surface du lymphocyte $T$ et des protéines associées CD4, CD8, CD2 et CD28. Les modules $C D 3$ et $\zeta 2$ représentent les modules de transduction autonomes décrits par A. M. Wegener et al. CD2 constitue une voie d'activation alternative qui interagit exclusivement avec le module $\zeta 2$.

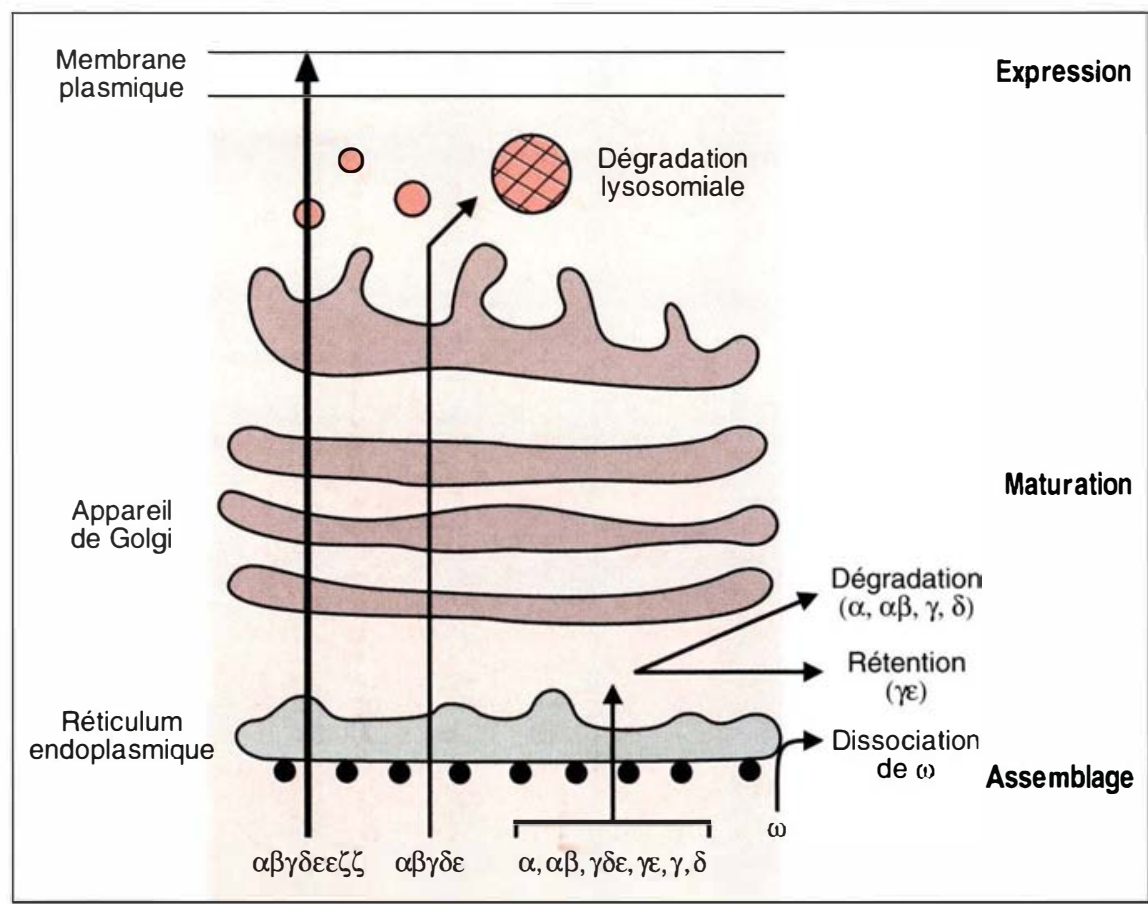

Figure 2. Parcours intracellulaire des complexes complets et partiellement assemblés. Les sites de dégradation proposés par J. D. Ashwell et R. D. Klausner sont indiqués. Les protéines s'associent dans le réticulum endoplasmique où la chaîne $\omega$ stabilise les complexes préformés, puis la maturation a lieu dans l'appareil de Golgi, aboutissant à l'expression, à la surface du lymphocyte $T$, des complexes entiers. transmembranaire de la protéine CD3- $\epsilon$.

Ces résultats suggéraicnt un épissage anormal. Nous avons donc séquencé des produits de PCR obtenus à partir de l'ADN génomique couvrant les deux sites d'épissage. Unc substitution hétérozygote $(\mathrm{T} \rightarrow \mathrm{C}$ ), au niveau du site d'épissage "GT " en 5' de l'intron 7, a été mise en évidence chez le patient et chez sa mère. Les mutations affectant ce site particulier d'épissage réduisent l'épissage normal mais ne l'empêchent pas complètement [14]. Dans le cas présent, nous pouvons mettre en évidence, en plus du produit où l'exon 7 est absent, un produit normalement épissé en très faible quantité. Ce produit de taille normalc permet d'expliquer l'expression membranaire résiduclle, détectéc au cytofluorimètre, chez le patient.

L'existence de cette mutation hétérozygote, partagéc avec la mère (dont les lymphocytes $\mathrm{T}$ sont phénotypiquement normaux et fonctionnels), suggérait qu'une autre mutation devait être présente chez le patient. Lors de l'étude de l'expression du gène CD3- $\epsilon$ chez le patient et chez ses parents, nous avons pu montrer une diminution de $50 \%$ de la quantité de messagers codant pour la chaînc CD3- $\epsilon$, chez le patient et chez son père. Ce résultat plaidait en faveur de l'existence d'une autre mutation, héritéc du pèrc, responsable soit d'un taux de transcription plus faible, soit de l'instabilité du messager. Nous avons séquencé les régions promotrices et enhancers de ce gène et réalisé une expérience d'élongation des chaînes naissantes d'AR N sur noyaux isolés (run on), pour éliminer l'hypothèse d'unc transcription anormale, et nous n'avons pas pu mettre en évidence de délétions ou de mutations. C'est lors du séquençage de l'ADN génomique que nous avons mis en évidence une substitution hétérozygote $(\mathrm{G} \rightarrow \mathrm{A})$ dans l'exon 6, produisant un codon stop. Cette mutation a été retrouvéc chez le patient et chez son père. Ici, la position précoce en 5 ' de ce codon stop aboutit vraiscmblablement à l'instabilité du transcrit et à sa rapide dégradation.

Le cas que nous avons étudié [15], où deux mutations affectent la chaîne 
CD3- $\epsilon$, met en évidence le rôle crucial de la chaîne CD3- $\epsilon$ au sein du complexe récepteur T/CD3. Le faible taux d'expression provient le plus probablement d'un récepteur T/CD3 complet où la chaîne CD3- $\epsilon$, produit de l'épissage normal de l'allèle maternel muté, est présente. Les signaux transmis via le récepteur pour l'antigène jouent un rôle déterminant dans la sélection (répertoire) et la maturation des progéniteurs $\mathrm{T}$ dans le thymus. Malgré le faible taux d'expression du récepteur $\mathrm{T}$ chez le patient, l'ontogénie des cellules $\mathrm{T}$ semble normale si l'on considère l'usage des familles de segments variables $\mathrm{V} \beta$ par la chaîne $\beta$ du TCR, ou le nombre de cellules $\mathrm{CD}^{+}{ }^{+}$ou $\mathrm{CD}^{+}$. Cela permet de penser qu'un faible nombre de récepteurs à la surface de la cellule $\mathrm{T}$ est suffisant pour l'ontogenèse thymique. In vitro, les lymphocytes $\mathrm{T}$ sanguins ne sont stimulables ni par des lectines ni par des anticorps anti-CD3 et anti-CD2, bien que les cellules $T$ expriment un nombre normal de molécules CD2 à la surface. Cela confirme qu'un nombre normal de récepteurs T/CD3 est indispensable pour que les ligands de CD2 puissent activer le lymphocyte $T$. Une prolifération partielle peut être restaurée par costimulation avec des anti-
CD3 et anti-CD28. Paradoxalement, les cellules du patient proliferent en présence des antigènes : Candida albicans ou anatoxine tétanique et en présence de cellules allogéniques [16]. Ces données montrent que l'activation des lymphocytes $\mathrm{T}$ peut être induite par la mise en jeu d'un faible nombre de récepteurs, à condition sans doute que le signal soit amplifié par des corécepteurs comme CD28.

Il existe un deuxième cas de défaut d'expression du TCR/CD3 observé au cours d'un déficit immunitaire héréditaire résultant de deux mutations affectant le gène codant pour CD3- $\gamma$ [17]. Il s'agit de deux mutations différentes : l'une est une mutation du codon d'initiation et l'autre, une mutation d'un site d'épissage. Ces deux mutations ne permettent pas la production de protéine CD3- $\gamma$. L'absence de cette protéine diminue mais n'abolit pas l'association normale ni l'expression à la surface du récepteur T/CD3. L'analyse en cytofluorimétrie des lymphocytes sanguins révèle environ $50 \%$ d'expression du TCR/CD3 à la surface. Au sein de ces complexes immunoprécipités, la chaîne CD3- $\gamma$ est absente. De plus, ces auteurs ont pu montrer que la chaîne CD3- $\gamma$ n'est pas indispensa- ble pour assurer la fonction helper [8] ; à l'opposé, la fonction et le développement des lymphocytes $\mathrm{T}$ cytotoxiques sont altérés. Ces données leur ont permis de proposer un modèle où les molécules CD4 et CD8 interagissent avec le complexe récepteur T/CD3 respectivement via les chaînes CD3- $\delta$ et CD3- $\gamma$.

Toute notre connaissance concernant lc récepteur pour l'antigène de la cellule repose essentiellement sur l'étude des mutants expérimentaux. L'étude de ces variants TCR/CD3, observés en pathologie humaine, permet non seulement d'élucider les mécanismes moléculaires responsables de ces déficits, mais permet aussi de valider et de compléter les résultats obtenus, grâce aux variants expérimentaux, sur l'assemblage, la stoechiométrie et l'expression du récepteur T/CD3

\section{Claire Soudais \\ Barbara Lisowska-Grospierre \\ Inserm U.132, hôpital Necker - Enfants- Malades, 149, rue de Sères, 75743 Paris Cedex 15, France}

\section{TIRÉS A PART}

C. Soudais.

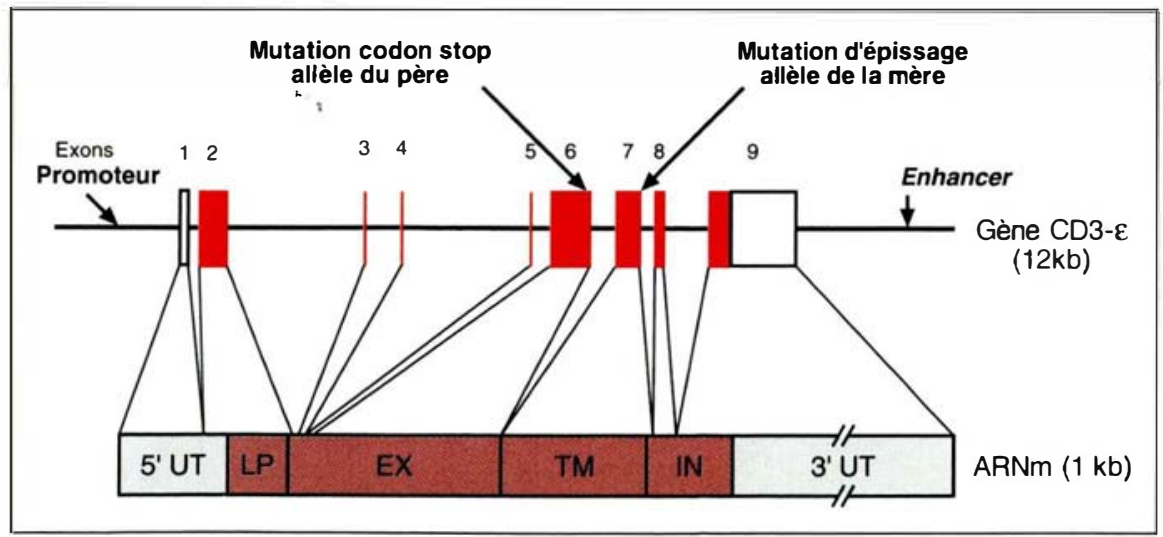

Figure 3. Schéma du gène et du messager CD3- - . La mutation $G \rightarrow A$ qui crée un codon stop se trouve dans l'exon 6, elle ségrège avec l'allèle hérité du père. La mutation $T \rightarrow C$ qui affecte le site d'épissage en $3^{\prime}$ de l'exon 7 codant pour la partie transmembranaire de la protéine ségrège avec l'allèle hérité de la mère. $5^{\prime}$ UT : partie $5^{\prime}$ non traduite ; LP: peptide signal; Ex : région extracellulaire ; TM : région transmembranaire ; IN : région intracellulaire ; $3^{\prime} U T$ : partie $3^{\prime}$ non traduite.

\section{RÉFÉRENCES}

1. Klausner RD, Lippincott-Schwartz J, Bonifacino JS. The T-cell antigen receptor : insight into organelle biology. Annu Rev Cell Biol $1990 ; 6: 403-31$.

2. Clevers $\mathrm{H}$, Alarcon $\mathrm{B}$, Wileman $\mathrm{T}$, Terhorst C. The T-cell receptor/CD3 complex : a dynamic protein ensemble. Annu Rev Immunol $1988 ; 6$ : 629-62.

3. Clayton LK, D'Adamio L, Howard FD, Sieh M, Hussey RE, Koyasu S, Reinherz

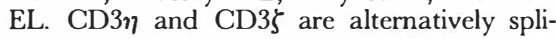
ced products of a common genetic locus and are transcriptionaly and/or post-transcriptionaly regulated furing T-cell development. Proc Nat Acad Sci USA 1991; 88 : 5202-6.

4. Wegener AMK, Letourneur F, Hoeveler A, Brocker $T$, Luton $F$, Malissen B. The $T$ cell receptor/CD3 complex is composed of at least two autonomous transduction modules. Cell 1992 ; 68 : 83-95.

5. Ashwell JD, Klausner RD. Genetic and mutational analysis of the T-cell antigen receptor. Annu Rev Immunol $1990 ; 8$ : 139-67. 
6. Blumberg RS, Ley S, Sancho J, Lonberg $\mathrm{N}$, Lacy E, McDermott F, Schad V, Greenstein JL, Terhorst C. Structure of the T-cell antigen receptor: evidence for two CD3- $\epsilon$ subunits in the T-cell receptor-CD3 complex. Proc Natl Acad Sci USA $1990 ; 87$ : 7220-4.

7. Jin YJ, Koyasu S, Moingeon P, Steinbrich R, Tarr GE, Reinherz FL. A fraction of CD3- $\epsilon$ subunits exists as disulfide-linked dimers in both human and murine $\mathrm{T}$ lymphocytes. J Biol Chem 1990; 265 : 15850-3.

8. Perez-Aciego P, Alarcon B, Arnaiz-Villena A, Terhorst C, Timon M, Segurado OG, Regueiro JR. Expression and function of a variant T-cell receptor complex lacking CD3- $\gamma$. J Exp Med 1991 ; 174 : 319-26.

9. Buferne $M$, Luton $F$, Letourneur $F$, Hoeveler A, Couez D, Barad M, Malissen B, Schmitt-Verhulst AM, Boyer C. Role of $\mathrm{CD} 3 \delta$ in surface expression of the TCR/CD3 complex and in activation for killing analysed with a CD3 $\delta$-negative cytotoxic Tlymphocyte variant. J Immunol $1992 ; 148$ : 657-64.

10. Wileman T, Carson GR, Concino M, Ahmed A, Terhorst C. The $\gamma$ and $\epsilon$ subunits of the CD3 complex inhibit pre-golgi degradation of newly synthesized T-cell antigen receptors. J Cell Biol 1990 ; 110 : 973-86.

11. Pettey CL, Alarcon B, Malin R, Weinberg $\mathrm{K}$, Terhorst C. T3-p28 is a protein associated with the $\delta$ and $\epsilon$ chains of the T-cell receptor-T3 antigen complex during biosynthesis. J Biol Chem 1987 ; 262 : 4854-9.

12. Alarcon B, Berkhout B, Breitmeyer J, Terhorst C. Assembly of the human T-cell Receptor-CD3 complex takes place in the endoplasmic reticulum and involves intermediary complexes between the CD3- $\gamma, \delta, \epsilon$ corc and single T-cell receptor $\alpha$ and $\beta$ chains. $J$ Biol Chem 1988 ; 263 : 2953-61.

13. Thoenes G, Soudais C, Le Deist F, Griscelli C, Fischer A, Lisowska-Grospierre B. Structural analysis of low TCR/CD3 complex expression in $\mathrm{T}$ cells of an immunodeficient patient. J Biol Chem 1992 ; 267 : 487-93.

14. Aebi M, Horning H, Padgett RA, Reiser J, Weissmann C. Sequence requirements for splicing of higher eukaryotic nuclear premRNA. Cell $1986 ; 47$ : 555-65.

15. Soudais C, de Villartay JP, Le Deist F, Fischer A, Lisowska-Grospierre B. Independent mutations of the human CD3- $\epsilon$ gene resulting in a T-cell receptor/CD3 complex immunodeficiency. Nature Genetics. 1993 ; 3 : $77-81$.

16. Le Deist F, Thoenes G, Corado J, Lisowska-Grospierre B, Fischer A. Immunodeficiency with low expression of the T-cell receptor/CD3 complex. Effect on T lymphocytes activation. Eur J Immunol 1991; 21 : 1641-7.

17. Arnaiz-Villena A, Timon M, Corell A, Perez-Aciego P, Martin-Villena JM, Regueiro JR. Primary immunodeficiency caused by mutations in the gene encoding the CD3- $\gamma$ subunit of the T-lymphocyte receptor. $N$ Engl $J$ Med 1992 ; 327 : 529-33.

$\mathrm{m} / \mathrm{s} n^{\circ} 6-7$ vol. 9, juin-juillet 93 